JOURNAL OF THE

AMERICAN MATHEMATICAL SOCIETY

Volume 10, Number 1, January 1997, Pages 123-138

S 0894-0347(97)00218-X

\title{
HOLOMORPHIC CHAINS AND THE SUPPORT HYPOTHESIS CONJECTURE
}

\author{
H. ALEXANDER
}

\section{INTRODUCTION}

Let $\Omega$ be a complex manifold and let $V$ be a (holomorphic) subvariety of $\Omega$ of pure (complex) dimension $k$. Then integration over $V$ defines a closed current of dimension $2 k$ in $\Omega$, denoted by $[V]$. More generally, let $\left\{V_{j}\right\}$ be a locally finite family of irreducible holomorphic subvarieties of $\Omega$ of pure dimension $k$ and let $\left\{n_{j}\right\}$ be integers. Then $T=\sum n_{j}\left[V_{j}\right]$ is a $2 k$ - current in $\Omega$-these are the holomorphic $k$-chains in $\Omega$.

Holomorphic chains are particular examples of the locally rectifiable currents of H. Federer [F]. A locally rectifiable s-current $T$ in an open subset $\Omega$ of $\mathbb{R}^{n}$ can be described as follows. There is a locally $\left(\mathcal{H}^{s}, s\right)$-rectifiable set $B$ in $\Omega$ and an $s$-vector field $\eta$ on $B$ which is locally $\mathcal{H}^{s}$-integrable over $B$ such that for $\mathcal{H}^{s}$-almost all $x \in B$, $\eta(x)$ is a simple $s$-vector that represents the approximate tangent space to $B$ at $x$ and $\|\eta(x)\|$ is a positive integer - the multiplicity of $T$ at $x$. Then the $s$-current $T$ is given by $T(\phi)=\int_{B}\langle\phi, \eta\rangle d \mathcal{H}^{s}$ for all $s$-forms $\phi$ with compact support in $\Omega$. In $\mathbb{C}^{n}$, this description can be refined. The $s$-forms can be decomposed into sums of $(a, b)$ forms with $a+b=s$. A $2 k$-current has bidimension $(k, k)$ if $T(\phi)=0$ for all forms $\phi$ of type $(a, b)$ with $(a, b) \neq(k, k)$. If $T$ has bidimension $(k, k)$ it follows that the approximate tangent space to $B$ is for $\mathcal{H}^{2 k}$-almost all $x \in B$ a complex $k$-dimensional linear space. If the natural orientation of this complex linear space agrees with the orientation induced by $\eta(x)$ for $\mathcal{H}^{2 k}$-almost all $x \in B$, one says that the $(k, k)$ current $T$ is positive. The space of all locally rectifiable $(k, k)$ currents on $\Omega$ is denoted by $\mathcal{R}_{(k, k)}^{\text {loc }}(\Omega)$.

Thus every holomorphic $k$-chain is a closed locally rectifiable $(k, k)$ current. We shall consider the converse. The first result in this direction was due to King $[\mathrm{K}]$. We denote by $\Omega$ an open subset of $\mathbb{C}^{n}$-however, being local, all of the results discussed below hold on complex manifolds.

King's Theorem. Let $T \in \mathcal{R}_{(k, k)}^{\text {loc }}(\Omega)$ be positive with $d T=0$. Then $T$ is a holomorphic k-chain.

There are some natural problems, discussed by Harvey and Shiffman [HS], in which one wants to apply a theorem of this type - especially when $T$ is a difference of elements of $\mathcal{R}_{(k, k)}^{\text {loc }}(\Omega)$ - but for which the positivity hypothesis is not satisfied.

Received by the editors July 15, 1994 and, in revised form, April 26, 1996.

1991 Mathematics Subject Classification. Primary 32C30, 32C25.

Key words and phrases. Holomorphic chains, currents of type $(k, k)$, locally rectifiable currents.

(C) 1997 American Mathematical Society 
This difficulty was overcome by the following extension of King's theorem. Here supp $T$ is the support of $T$ in $\Omega$.

Harvey-Shiffman Theorem. Let $T \in \mathcal{R}_{(k, k)}^{\text {loc }}(\Omega)$ with $d T=0$. Suppose that $\mathcal{H}^{2 k+1}(\operatorname{supp} T)=0$. Then $T$ is a holomorphic $k$-chain.

Harvey and Shiffman derived many nice applications of this theorem [HS]. They conjectured that the "support hypothesis" $\left(\mathcal{H}^{2 k+1}(\operatorname{supp} T)=0\right)$ could be dropped - they were able to weaken the hypothesis somewhat. More recently Shiffman $[\mathrm{S}]$ succeeded in verifying the conjecture in the hypersurface case, $k=n-1$. Our main result is that the conjecture is true in general.

Theorem. Let $T \in \mathcal{R}_{(k, k)}^{\text {loc }}(\Omega)$ with $d T=0$. Then $T$ is a holomorphic $k$-chain.

Our proof differs from that of Harvey and Shiffman who utilize the PoincaréLelong formula to treat the hypersurface case and then reduce to that by projections. We treat the case $k=1$ first. This has several advantages: (i) To handle the putative 1-variety which is the support of $T$, we can use the techniques of uniform algebras, which - especially in the hands of Errett Bishop [B], [W] - have proved to be powerful tools in treating 1-dimensional varieties, (ii) we can use, in the $k=1$ case, Federer's structure theorem for integral 1-currents - there is no hope for such a structure theorem in higher dimensions - and (iii) we can then use induction on $k$ starting from the case $k=1$ together with the nice slicing properties of rectifiable currents.

The primary reference for rectifiable currents is of course Federer's treatise on geometric measure theory $[\mathrm{F}]$. Harvey and Shiffman [HS] give an exposition of this material as it applies to complex analysis - we shall assume that the reader is familiar with their discussion and give references to $[\mathrm{F}]$ in those instances when we go beyond it. A nice exposition of the role of currents in complex analysis has been given by Harvey $[\mathrm{H}]$. An especially transparent sketch of the proof of the Harvey-Shiffman theorem is given in the survey paper of Harvey and Lawson [HL]. Shiffman [S] discusses the history of the support hypothesis conjecture. Finally I would like to thank the referee for some helpful comments.

\section{A Cauchy formula}

We shall use $b T$ to denote the boundary of a current $T$, defined by $b T(\phi)=T(d \phi)$. This is denoted by $\partial T$ in $[\mathrm{F}]$ but, following the custom in complex analysis, we shall reserve the use of $\partial$ for the complex exterior derivative which maps $(p, q)$-forms to $(p+1, q)$ - forms. Note that $b T$ and $d T$ agree up to a sign. Let $T \in \mathcal{R}_{(1,1)}^{\text {loc }}(\Omega)$ with $d T=0$ (and therefore $b T=0)$. We begin by establishing a Cauchy formula for $T$. Let $B(a, r)$ denote the ball of center $a$ and radius $r$. Suppose that the closure of $B(a, r)$ is contained in $\Omega$. Let $T(r)$ be the current $T\llcorner B(a, r)$. Except for a set of $r$ of $\mathcal{L}^{1}$-measure zero, the slice $b T(r)$ exists and is a closed rectifiable 1-current supported on $b B(a, r)$. Thus $b T(r)$ is a closed 1-dimensional integral currentthese have a nice characterization [F, 4.2.25]. Namely, there exists a countable family of rectifiable (oriented) Jordan curves $\left\{\gamma_{j}\right\}$ contained in $b B(a, r)$ such that $b T(r)=\sum\left[\gamma_{j}\right]$ and $M(b T(r))=\sum M\left(\left[\gamma_{j}\right]\right)$, where of course the mass $M\left(\left[\gamma_{j}\right]\right)$ is the length of $\gamma_{j}$. At this point we cannot preclude the possibility that the union $\Gamma$ of the $\left\{\gamma_{j}\right\}$ might be dense in $b B(a, r)$. The measure $\|b T(r)\|$ is the restriction of $\mathcal{H}^{1}$ to $\Gamma$ with multiplicity; i.e., $\|b T(r)\|=\sum_{j} \mathcal{H}^{1} \mid \gamma_{j}$. We consider the Cauchy 
transform of the 1-current $b T(r)$ in the coordinate function $z_{n}$. Since $\|b T(r)\|$ is a finite measure it follows that

$$
b T(r)\left(\left|d z_{n}\right| /\left|z_{n}-\alpha\right|\right) \equiv \sum_{j} \int_{\gamma_{j}}\left|d z_{n}\right| /\left|z_{n}-\alpha\right|<\infty
$$

for $\mathcal{L}^{2}$-almost all $\alpha$ in $\mathbb{C}$. Indeed, as a function of $\alpha$, this is locally integrable in $\mathbb{C}$, by an application of Fubini's theorem, (see [W], Lemma 2.4).

Let $\pi$ be the projection to the $n \underline{\text { th }}$ coordinate $\pi(z)=z_{n}$. For almost all $\alpha$, the slice $\langle T(r), \pi, \alpha\rangle$ exists and is a rectifiable 0 -current, i.e., this slice given by $\sum_{1}^{s} n_{j}\left[w_{j}\right]$ where the $w_{j}$ are distinct points in $B(a, r)$ with $\pi\left(w_{j}\right)=\alpha$ and the $n_{j}$ are non-zero integers (possibly $s=0$ and $\langle T(r), \pi, \alpha\rangle=0$ ) (see $[\mathrm{F}, 4.3 .8]$ ).

Cauchy Formula. Suppose that the slice $b T(r)$ exists as a closed rectifiable 1current supported on $b B(a, r)$. Let $\alpha \in \mathbb{C}$. Suppose that

$$
b T(r)\left(\left|d z_{n}\right| /\left|z_{n}-\alpha\right|\right)<\infty
$$

and that

$$
\text { the slice }\langle T(r), \pi, \alpha\rangle \text { exists and equals } \sum_{j=1}^{s} n_{j}\left[w_{j}\right] \text {. }
$$

Then for all polynomials $f$ in $\mathbb{C}^{n}$

$$
b T(r)\left(\frac{f d z_{n}}{z_{n}-\alpha}\right)=2 \pi i\langle T(r), \pi, \alpha\rangle(f)=2 \pi i \sum_{j=1}^{s} n_{j} f\left(w_{j}\right) .
$$

Remark. The integral representing $b T(r)\left(f d z_{n} /\left(z_{n}-\alpha\right)\right)$ converges (absolutely) by (1).

Proof. Set $u(z)=\left|z_{n}-\alpha\right|$. By $([\mathrm{F}], 4.2 .1$ and 4.3.6) for almost all $\epsilon>0$ the slice $\langle T(r), u, \epsilon\rangle$ exists, is rectifiable and we have

$$
\langle T(r), u, \epsilon\rangle=-b(T(r)\llcorner\{z: u(z)>\epsilon\})+(b T(r))\llcorner\{z: u(z)>\epsilon\}
$$

and

$$
\langle T(r), u, \epsilon\rangle=b(T(r)\llcorner\{z: u(z)<\epsilon\})-(b T(r))\llcorner\{z: u(z)<\epsilon\} .
$$

Set $S(\epsilon)=\langle T(r), u, \epsilon\rangle$ ( $r$ is fixed throughout this proof); $S(\epsilon)$ is a rectifiable 1current for almost all $\epsilon$. Let $\phi=f d z_{n} /\left(z_{n}-\alpha\right)$, a smooth (1,0)-form on the set $\{z: u(z) \geq \epsilon\}$. We apply (4) to $\phi$ and get

$$
S(\epsilon)\left[\frac{f d z_{n}}{z_{n}-\alpha}\right]=-T(r)\llcorner\{z: u(z)>\epsilon\}(d \phi)+(b T(r))\llcorner\{z: u(z)>\epsilon\}(\phi) .
$$

As $f /\left(z_{n}-\alpha\right)$ is holomorphic on $\{z: u(z)>\epsilon\}, d \phi$ is a $(2,0)$-form. Since $T$ has bidimension (1,1), $T(r)\llcorner\{z: u(z)>\epsilon\}$ is also a current of bidimension $(1,1)$. It follows that $T(r)\llcorner\{z: u(z)>\epsilon\}(d \phi)=0$. We get

$$
S(\epsilon)\left[\frac{f d z_{n}}{z_{n}-\alpha}\right]=(b(T(r))\llcorner\{z: u(z)>\epsilon\})(\phi) .
$$

It follows from hypothesis (1) and the dominated convergence theorem that the right-hand side of (6) converges to $b T(r)(\phi)$ as $\epsilon \rightarrow 0$. Thus to complete the proof of the Cauchy formula it remains to show that the left-hand side of (6) approaches $2 \pi i \sum n_{j} f\left(w_{j}\right)$ as $\epsilon \rightarrow 0$. 
To evaluate the left-hand side of (6) we use the fact that $\left(z_{n}-\alpha\right)\left(\bar{z}_{n}-\bar{\alpha}\right)=\epsilon^{2}$ on the support of $S(\epsilon)$ to get

$$
S(\epsilon)(\phi)=S(\epsilon)(\tau)
$$

where we set $\tau=\epsilon^{-2}\left(\bar{z}_{n}-\bar{\alpha}\right) f(z) d z_{n}$.

Then $d \tau=2 i \epsilon^{-2} f d x_{n} \wedge d y_{n}+\tau^{\prime}$, where $z_{n}=x_{n}+i y_{n}$ and $\tau^{\prime}$ is a (2,0)-form. Applying (5) we get

$$
S(\epsilon)(\tau)=(T(r)\llcorner\{z: u(z)<\epsilon\})(d \tau)-(b(T(r))\llcorner\{z: u(z)<\epsilon\})(\tau) .
$$

For the first term on the right-hand side of (7) we have

$$
\begin{aligned}
(T(r)\llcorner\{z: u(z)<\epsilon\})(d \tau)= & 2 i \epsilon^{-2}\left(T(r)\llcorner\{z: u(z)<\epsilon\})\left(f d x_{n} \wedge d y_{n}\right)\right. \\
& +\left(T(r)\llcorner\{z: u(z)<\epsilon\})\left(\tau^{\prime}\right) .\right.
\end{aligned}
$$

Here the second term on the right vanishes as above, since $\tau^{\prime}$ has type $(2,0)$. The first term can be written as the product of $2 \pi i$ and

$$
\left(\pi \epsilon^{2}\right)^{-1}\left(T(r)\llcorner\{z: u(z)<\epsilon\})\left(f d x_{n} \wedge d y_{n}\right) .\right.
$$

By the definition of slicing [F, 4.3.1] this last quantity approaches $\langle T(r), \pi, \alpha\rangle(f)=$ $\sum_{1}^{s} n_{j} f\left(w_{j}\right)$ as $\epsilon \rightarrow 0$. Finally we have, since $\epsilon^{-2}\left|\overline{z_{n}}-\bar{\alpha}\right| \leq\left(\left|z_{n}-\alpha\right|\right)^{-1}$ on the set $\{z: u(z)<\epsilon\}$

$$
\mid\left(b(T(r))\llcorner\{z: u(z)<\epsilon\})(\tau)|\leq M \cdot \| b T(r)| \mid\left\llcorner\{z: u(z)<\epsilon\}\left(\frac{1}{\left|z_{n}-\alpha\right|}\right)\right.\right.
$$

where $\mathrm{M}$ is the supremum of $|f|$ over $B(a, r)$. By hypothesis (1), the right-hand side converges to 0 as $\epsilon \rightarrow 0$. Thus, as $\epsilon \rightarrow 0$, the right-hand side of (7) approaches $2 \pi i \sum_{1}^{s} n_{j} f\left(w_{j}\right)$. This completes the proof of the Cauchy formula.

\section{RePRESENTING MEASURES}

For positive currents, lower bounds on the Hausdorff measure of the support can be obtained by an argument of Lelong (see $[\mathrm{K}]$ ). For the general case we shall need results on representing measures. For a general reference see Gamelin [G].

Proposition 1. Let $\mathcal{A}$ be the uniform algebra of functions holomorphic on the ball $B(a, r)$ in $\mathbb{C}^{n}$ and continuous on $\bar{B}(a, r)$. Let $\gamma$ be a rectifiable (open) Jordan arc in $b B(a, r)$. Let $\mu$ be a representing measure for $\mathcal{A}$ for a with support of $\mu$ contained in $b B(a, r)$. Then $\mu\left\llcorner\gamma<<\mathcal{H}^{1}\llcorner\gamma\right.$ and

$$
\frac{d \mu\llcorner\gamma}{d \mathcal{H}^{1}\llcorner\gamma} \leq \frac{1}{2 \pi r}
$$

Remark 1. This is an extension of a theorem of Errett Bishop [B] who proved it in the following setting: $V$ is a one-variety through the origin in a neighborhood of $\bar{B}(0, r)$ and $\mu$ is a representing measure for the origin supported on $b(V \cap B(0, r)(=$ a real analytic curve in $b B(0, r)$, except for a possible countable set of singularities) and $\gamma$ is $b(V \cap B(0, r))$. The proof which we give is essentially Bishop's - modified for our more general setting.

Remark 2. We do not assume any relationship between $\mu$ and $\gamma$. In particular the support of $\mu$ may be all of $b B(0, r)$. For example if $\mu$ is "normalized surface area" on $b B(0, r)$, then $d \mu\left\llcorner\gamma / d \mathcal{H}^{1}\llcorner\gamma=0\right.$ for every $\gamma$. 
Let $\Delta$ be the open unit disc in $\mathbb{C}$. For $0<\delta<\pi$, let $h_{\delta}=\mathcal{P}\left[\chi_{(-\delta, \delta)}\right]$ where $\mathcal{P}$ is the Poisson integral on $b \Delta$ and $\chi_{(-\delta, \delta)}$ is the characteristic function of $\left\{e^{i t}:-\delta<\right.$ $t<\delta\}$. Suppose that $g$ is a continuous real-valued function on the interval $(-a, a)$ with $a>0$ such that $g(-t)=g(t), g(0)=1,0<g(t)<1$ for $t \neq 0, g$ is decreasing for $t>0$ and $\lim _{t \rightarrow 0}(1-g(t)) / t=0$. Define a "tangential region" by

$$
R_{g}(\delta)=R(\delta)=\left\{\rho e^{i \phi}:-\delta \leq \phi \leq \delta \text { and } g(\phi) \leq \rho \leq 1\right\} .
$$

Lemma 1. Set, for $c>1, m(c, \delta)=\min \left\{h_{c \delta}(\zeta): \zeta \in R(\delta)\right\}$. For all $c>1$,

$$
\lim _{\delta \rightarrow 0+} m(c, \delta)=1 .
$$

Lemma 1 follows from a straightforward estimate of harmonic measure which we shall omit. Let $\mathbf{B}_{n}$ be the open unit ball centered at the origin in $\mathbb{C}^{n}$.

Lemma 2. Let $\left\{\gamma(t):-t_{0}<t<t_{0}\right\}$ be a continuous curve contained in $b \mathbf{B}_{n}$, $\gamma=\left(\gamma_{1}, \ldots, \gamma_{n}\right)$. Suppose that $\gamma(0)=e_{1}=(1,0, \ldots, 0)$, that $\gamma^{\prime}(0)$ exists and that the tangent to the curve is not complex tangential to $b \mathbf{B}_{n}$ at $e_{1}$, that is, $\gamma_{1}^{\prime}(0) \neq 0$. Then there exists a function $g$ as above such that for all $\delta_{0}>0, \gamma_{1}(t) \epsilon R_{g}\left(\delta_{0}\right)$ for all sufficiently small $t$.

Proof of Lemma 2. By the existence of the derivative, for small $t$ we can write

$$
\gamma_{1}(t)=1+i \lambda t+t \varepsilon(t)
$$

where $\lambda \neq 0$ is real and $\varepsilon(t)$ is continuous with $\varepsilon(0)=0$.

Writing $\varepsilon=\varepsilon_{1}+i \varepsilon_{2}$, we have

$$
1=|\gamma(t)|^{2} \geq\left|\gamma_{1}(t)\right|^{2}=1+2 t \varepsilon_{1}(t)+t^{2} \varepsilon_{1}(t)^{2}+t^{2}\left(\lambda+\varepsilon_{2}(t)\right)^{2} \geq 1+2 t \varepsilon_{1}(t) .
$$

It follows that

$$
0 \leq 1-\left|\gamma_{1}(t)\right| \leq 1-\left|\gamma_{1}(t)\right|^{2} \leq-2 t \varepsilon_{1}(t)
$$

We also have

$$
\left|\arg \left(\gamma_{1}(t)\right)\right|=\left|\arctan \left(\frac{\left(\lambda+\varepsilon_{2}(t)\right) t}{1+t \varepsilon_{1}(t)}\right)\right| \geq C|t|
$$

where $C$ is a positive constant. Set $q(t)=\sup \left\{\left|\epsilon_{1}(u)\right|:-|t| \leq u \leq|t|\right\}$. Then $q$ is continuous, $q(0)=0$ and $q$ is increasing in $|t|$. We have

$$
\left|\epsilon_{1}(t)\right| \leq q(|t|) \leq q\left(C^{-1}\left|\arg \left(\gamma_{1}(t)\right)\right|\right) .
$$

We get

$$
\left|\gamma_{1}(t)\right| \geq 1-2|t|\left|\epsilon_{1}(t)\right| \geq 1-2 C^{-1}\left|\arg \left(\gamma_{1}(t)\right)\right| q\left(C^{-1}\left|\arg \left(\gamma_{1}(t)\right)\right|\right) .
$$

This says that $\gamma_{1}(t) \epsilon R_{g}\left(\delta_{0}\right)$ for all sufficiently small $t$ if we take $g(t)=1-$ $2 C^{-1}|t| q\left(C^{-1}|t|\right)$. Clearly $\lim _{t \rightarrow 0}(1-g(t)) / t=0$, as required.

The next lemma clarifies a point of measure theory used in the proof of the proposition.

Lemma 3. Let $\alpha<<\beta$ be positive finite regular Borel measures on a compact space $X$. Let $u=d \alpha / d \beta$, the Radon-Nikodym derivative, which is defined $\beta$-almost everywhere. Suppose that $u \leq C \alpha$-almost everywhere. Then $u \leq C \beta$-almost everywhere. 
Proof. We have $u \leq C$ except on an $\alpha$-null set $Q$. Let $E$ be a Borel set. Then

$$
\alpha(E)=\alpha(E \backslash Q)=\int_{E \backslash Q} u d \beta \leq C \beta(E \backslash Q) \leq C \beta(E) .
$$

This gives the lemma.

Proof of Proposition 1. By a change of scale and a translation, we may assume that $r=1$ and $a=0$. Thus $\mathcal{A}$ is the ball algebra on $\mathbf{B}_{n}$. First we show that $\mu\left|\gamma<<\mathcal{H}^{1}\right| \gamma$. Let $K$ be a compact subset of $\gamma$ such that $\mathcal{H}^{1}(K)=0$. By a theorem of Davie and Øksendal ( [DO], corollary to Theorem 2), $K$ is a peak set for $\mathcal{A}$. It follows (see Rudin $[\mathrm{R}]$ ) that $\mu(K)=0$. This gives the absolute continuity.

We need to show that the Radon-Nikodym derivative $d(\mu \mid \gamma) / d\left(\mathcal{H}^{1} \mid \gamma\right) \leq 1 / 2 \pi$ at $\mathcal{H}^{1} \mid \gamma$-almost all points of $\gamma$. The tangent to $\gamma$ exists $\mathcal{H}^{1} \mid \gamma$-almost everywhere on $\gamma$ and so $\mu$-almost everywhere. on $\gamma$. Moreover this tangent is not complex tangential to $b \mathbf{B}_{n} \mu$-a.e. on $\gamma$. To see this, let $K$ be a compact subset of $\gamma$ such that the tangent to $\gamma$ exists and is complex tangential to $b \mathbf{B}_{n}$ at every point of $K$. It follows, as in [A2, Lemma 2], that one can apply a result of Davie and Øksendal ([DO], Theorem 1) to conclude that $K$ is a peak set for $\mathcal{A}$. As before it follows that $\mu(K)=0$. Thus at $\mu$-almost all points of $\gamma$, the tangent to $\gamma$ exists and is not complex tangential. It will suffice, by Lemma 3, to show that the Radon-Nikodym derivative $d(\mu \mid \gamma) / d\left(\mathcal{H}^{1} \mid \gamma\right) \leq 1 / 2 \pi$ at such points. Fix such a point of $\gamma$. By a unitary change of coordinates, we may assume that the point is $e_{1}=(1,0, \ldots, 0)$. Let $\rho$ be the projection to the first coordinate, $\rho(z)=z_{1}$. We may assume that $\gamma=\gamma(t)$ is parametrized by arc length $t \in[-\sigma, \sigma]$ near $e_{1}$ such that $e_{1}=\gamma(0)$ and $\rho\left(\gamma^{\prime}(0)\right)=i \lambda$, where $0<\lambda \leq 1$, since the tangent is not complex tangential.

Consider the rectifiable curve $\gamma_{1}(t)=\rho \circ \gamma(t)$ in $\mathbb{C}$. Since $\gamma_{1}^{\prime}(0)=i \lambda \neq 0$, it follows from Lemma 2 that there exists a continuous function $g$ as above such that for $\delta_{0}>0, \gamma_{1}(t) \in R_{g}\left(\delta_{0}\right)$ for $\sigma$ sufficiently small. We write $R(\delta)$ for $R_{g}(\delta)$

Let $\nu=\rho_{*}(\mu)$, the "push forward" of $\mu$. Then $\nu$ is a representing measure for the origin for the disk algebra, supported on the closed unit disk. Let $c$ be such that $c>1$. Then for $\delta<\pi / c$ we have

$$
\frac{c \delta}{\pi}=h_{c \delta}(0)=\int h_{c \delta} d \nu \geq \int_{R(\delta)} h_{c \delta} d \nu \geq m(c, \delta) \nu(R(\delta)) .
$$

Therefore

$$
\frac{\nu(R(\delta))}{2 \delta} \leq \frac{c}{2 \pi m(c, \delta)}
$$

It follows from Lemma 1 that

$$
\limsup _{\delta \rightarrow 0+} \frac{\nu(R(\delta))}{2 \delta} \leq \frac{c}{2 \pi} .
$$

Since $c$ is an arbitrary number such that $c>1$ we have

$$
\limsup _{\delta \rightarrow 0+} \frac{\nu(R(\delta))}{2 \delta} \leq \frac{1}{2 \pi} .
$$

In the notation of the proof of Lemma 2 we have, for all $C$ with $C>\lambda$,

$$
\left|\arg \left(\gamma_{1}(t)\right)\right|=\left|\arctan \left(\frac{\left(\lambda+\varepsilon_{2}(t)\right) t}{1+t \varepsilon_{1}(t)}\right)\right| \leq C|t|
$$


for all sufficiently small $t$. It follows that $\gamma_{1}((-\delta, \delta)) \subseteq R_{g}(C \delta)$ for small $\delta$. Hence

$$
\frac{\mu(\gamma(-\delta, \delta))}{2 \delta} \leq \frac{\nu\left(R_{g}(C \delta)\right)}{2 C \delta} C .
$$

Taking the limsup as $\delta \rightarrow 0+$ gives

$$
\limsup _{\delta \rightarrow 0+} \frac{\mu(\gamma(-\delta, \delta))}{2 \delta} \leq \frac{C}{2 \pi} .
$$

Since $\lambda \leq 1$ and $C$ is an arbitrary number such that $C>\lambda$, we get

$$
\limsup _{\delta \rightarrow 0+} \frac{\mu(\gamma(-\delta, \delta))}{2 \delta} \leq \frac{1}{2 \pi} .
$$

This completes the proof of the proposition.

\section{Areas of projections}

It is well known that the $2 k$-volume of a $k$-dimensional holomorphic subvariety $V$ of $\mathbb{C}^{n}$ is equal to the sum of the $2 k$-volumes, counted with multiplicity, of the projections of $V$ to the complex coordinate $k$-planes. We need a generalization of this. For each subset $I$ of $\{1,2, \ldots, n\}$ with $k$ elements we denote the projection onto $\mathbb{C}^{k}$ given by the $k$ coordinates $z_{j}$ with $j \in I$ by $\pi_{I}$; that is, $\pi_{I}(z)=\left(z_{i_{1}}, z_{i_{2}}, \ldots, z_{i_{k}}\right)$ where $I=\left\{i_{1}, i_{2}, \ldots, i_{k}\right\}$ (in increasing order). We write $\omega=i / 2 \sum_{j=1}^{n} d z_{j} \wedge d \bar{z}_{j}$; $\omega^{k} / k !=\sum \omega_{I}$ where $\omega_{I}$ is the volume element on the $k$-plane spanned over $\mathbb{C}$ by $\left\{e_{i}: i \in I\right\}$.

Lemma 4. Let $W$ be a $\left(\mathcal{H}^{2 k}, 2 k\right)$-rectifiable set in $\mathbb{C}^{n}$. Suppose that the approximate tangent space to $W$ is a complex linear space of dimension $k$ at $\mathcal{H}^{2 k}$-almost all points of $W$. Then

$$
\mathcal{H}^{2 k}(W)=\sum_{I} \int_{\mathbf{C}^{k}} N\left(\pi_{I} \mid W, \lambda\right) d \mathcal{L}^{2 k} \lambda .
$$

Remark. Here $N$ is a multiplicity function: it counts the number of points in a fiber of the map $\pi_{I} \mid W$. See [F, 2.10.9].

Proof. By [F, 3.2.25], there exists an $\mathcal{H}^{2 k} \mid W$-measurable function $E$ with values in the simple $2 k$-vectors of unit norm such that for $\mathcal{H}^{2 k}$-almost all points $w$ in $W$, the complex tangent space to $W$ at $w$ is the linear space of real dimension $2 k$ represented by $E(w)$. Then, by Wirtinger's equality [F, 1.8.2],

$$
1=|E(w)|=\epsilon(w)\left\langle E(w), \omega^{k} / k !\right\rangle
$$

where $\epsilon(w)= \pm 1$, according to whether or not $E(w)$ assigns the natural orientation to the complex linear space. It follows that $\epsilon$ is a measurable function. Thus replacing $E$ by $\epsilon E$, we may assume, without loss of generality, that $\epsilon \equiv 1$.

Hence we get

$$
|E(w)|=\left\langle E(w), \omega^{k} / k !\right\rangle=\sum_{I}\left\langle E(w), \omega_{I}\right\rangle .
$$


We have $\left\langle E(w), \omega_{I}\right\rangle=$ ap $J_{2 k}\left(\pi_{I} \mid W\right)(w)$ for $\mathcal{H}^{2 k}$-almost all points $w$ in $W$, where ap $J_{2 k}$ is the approximate $2 k$-Jacobian, see [F, 3.2.1]. Thus we get

$$
\begin{aligned}
\mathcal{H}^{2 k}(W) & =\int_{W}|E| d \mathcal{H}^{2 k}=\sum_{I} \int_{W} \text { ap } J_{2 k}\left(\pi_{I} \mid W\right)(w) d \mathcal{H}^{2 k} w \\
& =\sum_{I} \int_{\mathbf{C}^{k}} N\left(\pi_{I} \mid W, \lambda\right) d \mathcal{L}^{2 k} \lambda .
\end{aligned}
$$

The last equality follows from [F, 3.2.20].

\section{THE LOWER BOUND FOR $k=1$}

Let $T \in \mathcal{R}_{(1,1)}^{\text {loc }}(\Omega)$ with $b T=0$. Suppose that $a \in \operatorname{supp} T$ and that $\bar{B}(a, r) \subseteq \Omega$. The main result in this section is the following lower bound.

Lemma 5. Suppose that the slice bT(r) exists. Then $M(b T(r)) \geq 2 \pi r$.

Recall the notation $\Gamma=\bigcup \gamma_{j}$ and $M(b T)=\sum M\left(\left[\gamma_{j}\right]\right)=\sum$ length $\left(\gamma_{j}\right)$.

The proof of Lemma 5 requires several other lemmas.

Lemma 6. Suppose that $\alpha$ satisfies (1) and (2) of the Cauchy formula. Suppose also that the slice $\langle T(r), \pi, \alpha\rangle=\sum_{1}^{s} n_{j}\left[w_{j}\right]$ is non-zero and set $w=w_{1}$. Then there exists a representing measure $\mu$ for $w$ for the uniform algebra $\mathcal{A}$ of functions holomorphic on the ball $B(a, r)$ and continuous on $\bar{B}(a, r)$ with supp $\mu \subseteq b B(a, r)$ and $\mu$ concentrated on $\Gamma$; i.e., $\mu(\Gamma)=1$.

Proof of Lemma 6. Choose a polynomial $g$ such that $g\left(w_{1}\right)=1$ and $g\left(w_{j}\right)=0$ for $j>1$. Then for every polynomial $f$ the Cauchy formula yields

$$
b T(r)\left(\frac{f g d z_{n}}{z_{n}-\alpha}\right)=2 \pi i \sum_{j} n_{j} f\left(w_{j}\right) g\left(w_{j}\right)=2 \pi i n_{1} f(w) .
$$

Hence $f(w)=\int_{\Gamma} f d \sigma$ where

$$
d \sigma=\left.\frac{1}{2 \pi i n_{1}} \sum_{j} \frac{g d z_{n}}{z_{n}-\alpha}\right|_{\gamma_{j}}
$$

Thus $\sigma$ is a complex representing measure for $w$ concentrated on $\Gamma$. It follows ([G], Theorem II.2.2, p.33) that there exists a (non-negative) representing measure $\mu$ for $w$ with $\mu<<\sigma$. Hence $\mu(\Gamma)=1$ as required.

Lemma 7. For all $\delta$ such that $0<\delta<r$ there exist $a w \in B(a, \delta)$ and a representing measure $\mu$ for $w$ for the uniform algebra $\mathcal{A}$ of functions holomorphic on the ball $B(a, r)$ and continuous on $\bar{B}(a, r)$ with supp $\mu \subseteq b B(a, r)$ and with $\mu$ concentrated on $\Gamma$; i.e., $\mu(\Gamma)=1$.

Proof of Lemma 7. We may assume that $a=0$. Recall, from the Introduction, that the 2-current $T$ is given by $T(\phi)=\int_{B}\langle\phi, \eta\rangle d \mathcal{H}^{2}$ for all 2-forms $\phi$ with compact support in $\Omega$. We set $W=B \cap B(0, \delta) ; W$ is a $\left(\mathcal{H}^{2}, 2\right)$-rectifiable set. Since $0 \in \operatorname{supp} T, \mathcal{H}^{2}(W)>0$. Hence by Lemma 4

$$
\sum_{k} \int_{\mathbf{C}} N\left(\pi_{k} \mid W, \lambda\right) d \mathcal{L}^{2} \lambda>0
$$

where $\pi_{k}$ is the projection to the $k \underline{\text { th }}$ coordinate given by $\pi_{k}(z)=z_{k}$. Without loss of generality we may assume that $\int N\left(\pi_{n} \mid W, \lambda\right) d \mathcal{L}^{2} \lambda>0$. 
To prove the lemma it will suffice, by Lemma 6 , to find an $\alpha$ satisfying (1) and (2) of the Cauchy formula such that the slice $\langle T(r), \pi, \alpha\rangle=\sum_{1}^{s} n_{j}\left[w_{j}\right]$ is non-zero and such that one of the points $w_{j}$ is contained in $B(0, \delta)$. Suppose that the slices $\left\langle T(r), \pi_{n}, \alpha\right\rangle$ and $\left\langle T(\delta), \pi_{n}, \alpha\right\rangle$ both exist-this is true for $\mathcal{L}^{2}$-almost all $\alpha$. By Federer's basic result on the slices of a rectifiable current [F, 4.3.8], it follows that $\left\langle T(r), \pi_{n}, \alpha\right\rangle \mid B(0, \delta)=\left\langle T(\delta), \pi_{n}, \alpha\right\rangle$ for $\mathcal{L}^{2}$-almost all $\alpha$. Since (1) and (2) hold for $\mathcal{L}^{2}$-almost all $\alpha$, it thus suffices to show the slice $\left\langle T(\delta), \pi_{n}, \alpha\right\rangle$ exists and is non-zero for a set of $\alpha$ of positive $\mathcal{L}^{2}$-measure.

We argue by contradiction and suppose that $\left\langle T(\delta), \pi_{n}, \alpha\right\rangle=0$ for $\mathcal{L}^{2}$-almost all $\alpha$. Define another current $T^{\prime} \in \mathcal{R}_{(1,1)}^{\text {loc }}$ by $T^{\prime}(\phi)=\int_{W}\langle\phi, \xi\rangle d \mathcal{H}^{2}$ for 2 -forms $\phi$, where $\xi(w)= \pm \eta(w)$ is a simple 2-vector which represents the naturally oriented complex tangent plane to $W$ at $w$ for $\mathcal{H}^{2}$-almost all points $w$ of $W$, the sign \pm being chosen so that $T^{\prime}$ is a positive current. The argument of Lemma 4 shows that $\xi$ is measurable and thus $T^{\prime}$ is a well-defined rectifiable current of bidimension $(1,1)$ with support in $\bar{B}(0, \delta)$; we cannot assert that $T^{\prime}$ is closed. It follows from [F, 4.3.8] that the slices $\left\langle T(\delta), \pi_{n}, \alpha\right\rangle$ and $\left\langle T^{\prime}, \pi_{n}, \alpha\right\rangle$, for $\mathcal{L}^{2}$-almost all $\alpha$, differ as 0 -currents only in the signs of the integral weights attached to the points of their supports; i.e., the supports of the two slices are equal for $\mathcal{L}^{2}$-almost all $\alpha$. From our assumption that $\left\langle T(\delta), \pi_{n}, \alpha\right\rangle=0$ for $\mathcal{L}^{2}$-almost all $\alpha$, it follows that $\left\langle T^{\prime}, \pi_{n}, \alpha\right\rangle=0$ for $\mathcal{L}^{2}$-almost all $\alpha$. Hence, by [F, 3.2.3, 4.1.30, 4.3.2(1)], we have

$$
\begin{aligned}
\int_{\mathbf{C}} N\left(\pi_{n} \mid W, \lambda\right) d \mathcal{L}^{2} \lambda & =\int_{W} J_{2}\left(\pi_{n} \mid W\right)(w) d \mathcal{H}^{2} w \\
& \leq T^{\prime}\left(d x_{n} \wedge d y_{n}\right) \\
& =\int_{\mathbf{C}}\left\langle T^{\prime}, \pi_{n}, \alpha\right\rangle(1) d \mathcal{H}^{2} \alpha=0 .
\end{aligned}
$$

This contradicts the fact that the first integral is strictly positive - the lemma follows.

Lemma 8. Let $\mu$ be a representing measure for a for the uniform algebra $\mathcal{A}$ of functions holomorphic on the ball $B(a, r)$ and continuous on $\bar{B}(a, r)$ with supp $\mu \subseteq$ $b B(a, r)$. Let $\left\{\gamma_{j}\right\}$ be a countable family (not necessarily distinct) of rectifiable Jordan curve in $b B(a, r)$. Then

$$
\sum_{j} \text { length }\left(\gamma_{j}\right) \geq 2 \pi r \mu\left(\bigcup \gamma_{j}\right)
$$

Proof. Applying Proposition 1 for fixed $j$ we get $2 \pi r \mu\left(\gamma_{j}\right) \leq \operatorname{length}\left(\gamma_{j}\right)$. Summing over $j$ we get

$$
2 \pi r \mu\left(\bigcup \gamma_{j}\right) \leq 2 \pi r \sum \mu\left(\gamma_{j}\right) \leq \sum \operatorname{length}\left(\gamma_{j}\right)
$$

Proof of Lemma 5. By Lemma 7, we can find $w$ arbitrarily close to $a$ and a representing measure $\mu^{\prime}$ for $w$ with $\mu^{\prime}$ concentrated on the union of the $\left\{\gamma_{j}\right\}$. Let $\Phi$ be a biholomorphism of the ball $B(a, r)$ which takes $w$ to $a$. Set $\mu=\Phi_{*}\left(\mu^{\prime}\right)$. Then $\mu$ is a measure representing $a$ and concentrated on the curves $\left\{\Phi\left(\gamma_{j}\right)\right\}$. By Lemma 8 we conclude that

$$
2 \pi r=2 \pi r \mu\left(\bigcup \Phi\left(\gamma_{j}\right)\right) \leq \sum \operatorname{length}\left(\Phi\left(\gamma_{j}\right)\right) .
$$


As $w$ approaches $a$, the biholomorphism $\Phi$ can be chosen to approach the identity map in the $\mathcal{C}^{2}$ topology. Hence the lengths of the $\left\{\Phi\left(\gamma_{j}\right)\right\}$ can be chosen arbitrarily close to the lengths of the $\left\{\gamma_{j}\right\}$. This gives Lemma 5 .

\section{Proof of the Theorem}

We shall first give a proof of the Theorem based on the Harvey- Shiffman theorem. After that we shall sketch a new proof which is independent of theirs. We proceed by induction on $k$.

First suppose that $T \in \mathcal{R}_{(1,1)}^{\text {loc }}(\Omega)$ with $b T=0$. Suppose that $a \in \operatorname{supp} T$ and that $\bar{B}(a, R) \subseteq \Omega$. We claim that $M(T(R)) \geq \pi R^{2}$. We have, by [F, 4.3.2], $M(T(R)) \geq \int_{0}^{R} M(b T(r)) d r$. By Lemma $5 M(b T(r)) \geq 2 \pi r$ for almost all $r<R$ and so the claim follows. From the claim it follows, by a general covering argument of measure theory [F, 2.10.19(3), 2.10.6], that the support of $T$ has locally finite $\mathcal{H}^{2}$-measure. This implies the support hypothesis and so by the Harvey-Shiffman theorem, $T$ is a holomorphic 1- chain.

Induction step. Suppose that the Theorem holds for elements of $\mathcal{R}_{(j, j)}^{\text {loc }}(D)$ for $D$ open in $\mathbb{C}^{n}$ with $j<k$. Let $T \in \mathcal{R}_{(k, k)}^{\text {loc }}(\Omega)$ with $b T=0$. We need to show that $T$ is a holomorphic $k$-chain. For $a \in \mathbb{C}^{n}$ and $\delta>0$ denote a polydisk by $\Delta=\Delta(a, \delta)=\left\{z \in \mathbb{C}^{n}:\left|z_{j}-a_{j}\right|<\delta, j=1,2, \ldots, n\right\}$. Suppose that $a \in \operatorname{supp} T$ and that $\bar{\Delta} \subseteq \Omega$.

Lemma 9. $\|T\|(\Delta) \geq C \delta^{2 k}$, where $C$ is a constant depending only on $n$.

Just as in the $k=1$ case this lower bound implies the support hypothesis and so the Harvey-Shiffman theorem can be applied to conclude that $T$ is a holomorphic $k$-chain.

For the proof of Lemma 9 we shall use the following fact (see [ATU]) about volumes of projections - the main point is that here the volumes are taken without multiplicity.

Lemma 10. Let $V$ be a subvariety of pure dimension $j$ of a ball of radius $r$ in $\mathbb{C}^{n}$ such that $V$ contains the center of the ball. Then

$$
\sum_{I} \mathcal{L}^{2 j}\left(\pi_{I}(V)\right) \geq C r^{2 j}
$$

Remark. Here $C$ is a constant depending only on $n$ and the projections $\pi_{I}$ to $\mathbb{C}^{j}$ are as in Lemma 4. As Lemma 4 shows, if the volumes of the projections are taken with multiplicity, then this sum equals $\mathcal{H}^{2 j}(V)$ which is known to be at least equal to $c_{j} r^{2 j}$, where $c_{j}$ is the $\mathcal{L}^{2 j}$-measure of the unit ball in $\mathbb{C}^{j}$.

Let $\pi_{j}(z)=z_{j}$, the projection to the $j \underline{\text { th }}$ coordinate.

Lemma 11. There exist a $j$ satisfying $1 \leq j \leq n$ and a measurable set $E \subseteq \mathbb{C}$ of positive $\mathcal{L}^{2}$-measure such that the slices $\left\langle T \mid \Delta(0, \delta / 4), \pi_{j}, \alpha\right\rangle$ exist and are non-zero for all $\alpha \in E$.

Proof of Lemma 11. As in the proof of Lemma 7, we define $T^{\prime}$ to be the positive $(k, k)$-current associated to $T \mid \Delta(0, \delta / 4)$. Since the supports of the slices of $T \mid \Delta(0, \delta / 4)$ and $T^{\prime}$ agree for $\mathcal{L}^{2}$-almost all $\alpha$ (see the proof of Lemma 7 ), it suffices to prove the lemma for $T^{\prime}$. For each $j$ we have by [F, 4.3.2(1)]

$$
I_{j} \equiv \int_{\mathbf{C}}\left\langle T^{\prime}, \pi_{j}, z_{j}\right\rangle\left(\omega^{k-1}\right) \frac{i}{2} d z_{j} \wedge d \bar{z}_{j}=T^{\prime}\left(\omega^{k-1} \wedge \frac{i}{2} d z_{j} \wedge d \bar{z}_{j}\right) .
$$


Summing over $j$ gives $\sum_{j} I_{j}=T^{\prime}\left(\omega^{k}\right)$. But since $0 \in \operatorname{supp} T$ it follows that $T^{\prime}\left(\omega^{k} / k !\right)=M\left(T^{\prime}\right)>0$. Hence $I_{j} \neq 0$ for some $j$ and this gives the lemma.

At this point it will be useful to note two of the functorial properties of slicing. Assume that $S \in \mathcal{R}_{(k, k)}^{\text {loc }}(\Omega)$ with $\Omega \subseteq \mathbb{C}^{n}, f: \Omega \rightarrow \mathbb{C}^{s}$ is a holomorphic map and that the slice $\langle S, f, z\rangle$ exists as a locally rectifiable $2 k$-current. Then (a) $d\langle S, f, z\rangle=$ $\langle d S, f, z\rangle$, see [HS, 1.3.9] (in particular if $d S=0$, then $d\langle S, f, z\rangle=0$ ) and (b) $\langle S, f, z\rangle$ is of bidimenson $(k-s, k-s)$ (this follows from the fact that for an $(a, b)$ form $\phi,\langle S, f, z\rangle(\phi)$ is defined as a limit of $S$ applied to certain $(a+s, b+s)$-forms). A consequence of these facts is that the induction hypothesis can be applied to conclude that almost all of the complex coordinate slices of $T$ are holomorphic chains (of the appropriate dimension).

Proof of Lemma 9. Without loss of generality we can assume that the $j$ in Lemma 11 is $n$. Now consider a slice $T_{\alpha} \equiv\left\langle T \mid \Delta(0, \delta), \pi_{n}, \alpha\right\rangle$. We may assume that these exist for all $\alpha \in E$ - the set given in Lemma 11. By the induction hypothesis, $T_{\alpha}$ is a holomorphic $(k-1)$-chain in $\Delta$. Hence the support is a pure $(k-1)$-dimensional subvariety $V_{\alpha}$ of $\Delta$. Moreover by Lemma 11 (where $j=n$ ), $V_{\alpha}$ contains a point of $\Delta(0, \delta / 4)$ for each $\alpha \in E$. A ball of radius $\delta / 4$ centered at such a point is contained in $\Delta(0, \delta / 2)$. Hence by Lemma 10 ,

$$
\sum_{I} \mathcal{L}^{2 k-2}\left(\pi_{I}\left(V_{\alpha} \cap \Delta(0, \delta / 2)\right)\right) \geq C \delta^{2 k-2}
$$

for all $\alpha \in E$ (the sum is for all projections $\pi_{I}$ to $\mathbb{C}^{k-1}$ ).

We write $T(\delta)=T \mid \Delta$. We apply next a basic "Fubini" type result of Federer on iterated slicing. By $[\mathrm{F}, 4.3 .5]$, "joint" slicing agrees almost everywhere with iterated slicing. Applying this twice, we have that the two iterated slices in both orders are equal almost everywhere. In our setting this means the following: Let $I$ be a subset of size $k-1$ of $\{1,2, \ldots, n-1\}$ and let $\pi_{I}: \mathbb{C}^{n} \rightarrow \mathbb{C}^{k-1}$ be the associated projection. Then, for $z^{\prime} \in \mathbb{C}^{k-1}$ and $\alpha \in \mathbb{C}$, we have

$$
\left\langle\left\langle T(\delta), \pi_{n}, \alpha\right\rangle, \pi_{I}, z^{\prime}\right\rangle=\left\langle\left\langle T(\delta), \pi_{I}, z^{\prime}\right\rangle, \pi_{n}, \alpha\right\rangle
$$

for $\mathcal{L}^{k}$-almost all $\left(z^{\prime}, \alpha\right) \in \mathbb{C}^{k}$. Let $N_{I}$ be the set of measure zero in $\mathbb{C}^{k}$ where this does not hold. Set $N_{0}=\bigcup_{I} N_{I}$, a set of measure zero. By Fubini's theorem, for $\mathcal{L}^{2}$-almost all $\alpha \in E, N_{0}(\alpha) \equiv\left\{z^{\prime} \in \mathbb{C}^{k-1}:\left(z^{\prime}, \alpha\right) \in N_{0}\right\}$ has $\mathcal{L}^{2 k-2}$-measure zero in $\mathbb{C}^{k-1}$. Fix such an $\alpha \in E$ for the remainder of the proof of Lemma 9.

Note that in (8) the terms in the sum involving $\pi_{I}$ with an $I$ containing $n$ are zero since $z_{n}$ is constant on $V_{\alpha}$. Hence we conclude from (8) that there exists a subset $I_{0}$ of size $k-1$ of $\{1,2, \ldots, n-1\}$ such that $\mathcal{L}^{2 k-2}\left(\pi_{I_{0}}\left(V_{\alpha} \cap \Delta(0, \delta / 2)\right)\right) \geq C \delta^{2 k-2}$, where $C$, in this proof, will denote a generic positive constant (depending only on $n)$. Without loss of generality we may assume that $I_{0}=\{1,2, \ldots, k-1\}$.

Let $S_{1}$ be the singular set of $V_{\alpha}$. Consider the the restriction of $\pi_{I_{0}}$ to $V_{\alpha} \backslash S_{1}$ call this smooth map $\rho$. Let $S_{2}$ be the set of singular values of $\rho$. Then $\rho\left(S_{1}\right) \cup S_{2}$ has $\mathcal{L}^{2 k-2}$-measure zero in $\mathbb{C}^{k-1}$. Define $P=\pi_{I_{0}}\left(V_{\alpha} \cap \Delta(0, \delta / 2)\right) \backslash\left(N_{0}(\alpha) \cup \rho\left(S_{1}\right) \cup S_{2}\right)$. Note that $\mathcal{L}^{2 k-2}(P)=\mathcal{L}^{2 k-2}\left(\pi_{I_{0}}\left(V_{\alpha} \cap \Delta(0, \delta / 2)\right)\right) \geq C \delta^{2 k-2}$.

If $z^{\prime} \in P$, there exists $z^{\prime \prime} \in \mathbb{C}^{n-(k-1)}$ such that $z \equiv\left(z^{\prime}, z^{\prime \prime}\right) \in V_{\alpha} \cap \Delta(0, \delta / 2)$. Hence $z \in \operatorname{supp} T_{\alpha}=V_{\alpha}$. Since $z$ is a regular point of $V_{\alpha}$ and $d \rho(z)$ has $\mathbb{C}$-rank $k-1$ (by the construction of $P$ ), it follows that $z \in \operatorname{supp}\left\langle T_{\alpha}, \pi_{I_{0}}, z^{\prime}\right\rangle$. Since $z^{\prime} \notin N_{0}(\alpha)$, it follows by (9) that $z \in \operatorname{supp}\left\langle\left\langle T(\delta), \pi_{I_{0}}, z^{\prime}\right\rangle, \pi_{n}, \alpha\right\rangle$. Hence $z \in$ $\operatorname{supp}\left\langle T(\delta), \pi_{I_{0}}, z^{\prime}\right\rangle$. Set $T\left(z^{\prime}\right)=\left\langle T(\delta), \pi_{I_{0}}, z^{\prime}\right\rangle$. Again by the induction hypothesis, 
$T\left(z^{\prime}\right)$ is a holomorphic 1-chain for almost all $z^{\prime} \in \mathbb{C}^{k-1}$. But since for $z^{\prime} \in P$ the support of $T\left(z^{\prime}\right)$ is a 1 -variety in $\Delta$ containing the point $z \in \Delta(0, \delta / 2)$ we argue as above (with a ball of radius $\delta / 2$ centered at $z$ ) to conclude that $\mathcal{H}^{2}\left(\operatorname{supp} T\left(z^{\prime}\right)\right) \geq$ $C \delta^{2}$ for $z^{\prime} \in P$. Hence $M\left(T\left(z^{\prime}\right)\right) \geq C \delta^{2}$ for $z^{\prime} \in P$. Set $\Delta^{\prime}=\left\{z^{\prime} \in \mathbb{C}^{k-1}:\left|z_{j}^{\prime}\right|<\right.$ $\delta, j=1,2, \ldots, k-1\}$. We now have by $[\mathrm{F}, 4.3 .2]$

$$
\begin{aligned}
M(T(\delta)) & \geq \int_{\Delta^{\prime}} M\left(T\left(z^{\prime}\right)\right) d \mathcal{L}^{2 k-2} z^{\prime} \geq \int_{P} M\left(T\left(z^{\prime}\right)\right) d \mathcal{L}^{2 k-2} z^{\prime} \\
& \geq C \delta^{2} \mathcal{L}^{2 k-2}(P) \geq C \delta^{2 k} .
\end{aligned}
$$

This completes the proof of Lemma 9 .

\section{Alternate proof of the Theorem}

We shall now indicate a proof of the Theorem which does not appeal to the Harvey-Shiffman theorem. This yields an independent proof of the Theorem that is perhaps shorter, in part, because we are able to use induction.

Let $T \in \mathcal{R}_{(k, k)}^{\text {loc }}(\Omega)$ with $d T=0$. Let $X=\operatorname{supp} T$. By a general property of flat currents (cf. [HS, Theorem 1.9]), it suffices to show that $X$ is locally contained in a $k$-dimensional subvariety of $\Omega$. Let $a \in X$. We show that $X$ is contained in a subvariety near $a$. Without loss of generality, $a=0$.

As before we let $\pi_{I}$ be the projection to $\mathbb{C}^{k}$ associated to a subset $I$ of $\{1,2, \ldots, n\}$ of size $k$. Assume for the moment that $X$ has locally finite $\mathcal{H}^{2 k}$-measure. It follows that, after a possible linear change of coordinates, each of the coordinate planes $\left\{z: \pi_{I}(z)=0\right\}$ meets $X$ in a discrete set at 0 (see [S2]). Fix $I_{0}=\{1,2, \ldots, k\}$ and write $\pi$ for $\pi_{I_{0}}$. There exist $r>0$ and $\delta>0$ such that $X$ is disjoint from $\bar{\Delta}^{\prime}(r) \times b \Delta^{\prime \prime}(\delta)$ where $\Delta^{\prime} \equiv \Delta^{\prime}(r)=\left\{z^{\prime} \in \mathbb{C}^{k}:\left|z_{j}^{\prime}\right|<r, 1 \leq j \leq k\right\}$ and $\Delta^{\prime \prime} \equiv$ $\Delta^{\prime \prime}(\delta)=\left\{z^{\prime \prime} \in \mathbb{C}^{n-k}:\left|z_{j}^{\prime \prime}\right|<\delta, 1 \leq j \leq n-k\right\}$. Set $\Delta=\Delta^{\prime} \times \Delta^{\prime \prime}$ and $X_{0}=X \cap \Delta$. Then $\pi \mid X_{0}$ is a proper map from $X_{0}$ to $\Delta^{\prime}$ and, writing $z=\left(z^{\prime}, z^{\prime \prime}\right)$, the projection $\pi$ to $\mathbb{C}^{k}$ is given by $\pi(z)=z^{\prime}$. As usual, $\pi_{j}: \Delta \rightarrow \mathbb{C}$ and $\pi_{j}^{\prime}: \Delta^{\prime} \rightarrow \mathbb{C}$ where $\pi_{j}(z)=z_{j}$ and $\pi_{j}^{\prime}\left(z^{\prime}\right)=z_{j}^{\prime}$ denote the projections of $\Delta$ and $\Delta^{\prime}$ to (the $j$ th coordinate in) $\mathbb{C}$. Entirely analogous statements can be made for each of the other projections $\pi_{I}$.

Lemma 12. $X$ (= the support of $T$ ) has locally finite $\mathcal{H}^{2 k}$-measure. For coordinates and the polydisk $\Delta$ chosen as above, there exists a $k$-dimensional subvariety $V$ of $\Delta$ such that for $1 \leq j \leq k$, and for $\mathcal{L}^{2}$-almost all $\alpha \in\{\lambda \in \mathbb{C}:|\lambda|<r\}$, the slice $\left\langle T \mid \Delta, \pi_{j}, \alpha\right\rangle$ exists, is a holomorphic $(k-1)$-chain in $\Delta$ and its support is contained in $V$.

Remark 1. The slices $\left\langle T \mid \Delta, \pi_{j}, \alpha\right\rangle$ exist (this means as rectifiable $(k-1, k-1)$ currents!) for almost all $\alpha$.

Remark 2. Lemma 12 is stated in the geometry of the projection $\pi_{I_{0}}$. Of course a corresponding result holds for each projection $\pi_{I}$. We denote the corresponding $k$-dimensional variety at 0 by $V_{I}$. We set $\mathcal{V}=\bigcup_{I} V_{I}$.

We shall prove the conjunction of our Theorem and Lemma 12 by induction on $k$. The scheme of the proof is this: (i) we show Lemma 12 for $k=1$; and then (ii) the Theorem for $k=1$; (iii) for $k>1$, assuming the Theorem for $j<k$, we prove Lemma 12 for $k$; (iv) we deduce the Theorem for $k$ from Lemma 12 for $k$.

Note that steps (ii) and (iv) are essentially the same; that is, each says that, for a particular $k$, Lemma 12 implies the Theorem. We prove this first. Recall that to 
prove the Theorem it suffices to show that $X$ is locally contained in a $k$-dimensional subvariety near $0 \in X$. It follows from Lemma 12 and Remark 2 following it that if $\mathcal{N}$ is a ball about 0 in $\Delta$ of sufficiently small radius $\rho$, then for each $j, 1 \leq j \leq n$, the support of the slice $\left\langle T \mid \mathcal{N}, \pi_{j}, \alpha\right\rangle$ is contained in $\mathcal{V}$ for almost all $\alpha$ such that $|\alpha|<\rho$.

Let $p \in X \cap \mathcal{N}$ and take a small ball about $p$. Then, by Lemma 11 ( with 0 translated to $p$ ), we conclude that any such ball contains a point of $\mathcal{V}$. Hence, as $\mathcal{V}$ is closed, it follows that $X \cap \mathcal{N} \subseteq \mathcal{V}$. This shows that the support of $T$ is locally contained in the $k$-dimensional variety $\mathcal{V}$ and the theorem follows.

Step (i). We prove Lemma 12 for $k=1$. We have $T \in \mathcal{R}_{(1,1)}^{\text {loc }}(\Omega)$ with $d T=0$. Let $X=\operatorname{supp} T$. It follows as before from Lemma 5 that $X$ has locally finite $\mathcal{H}^{2}$ measure. Let $\rho(z)=\left|z^{\prime}\right|$ where we write $z=\left(z^{\prime}, z^{\prime \prime}\right) \in \mathbb{C} \times \mathbb{C}^{n-1}$. Shrinking $r$ we may assume that the slice $b T(r) \equiv\left\langle T \mid \Omega \cap\left(\mathbb{C} \times \Delta^{\prime \prime}(\delta)\right), \rho, r\right\rangle$ exists as a rectifiable 1-current supported in $K \equiv X \cap\left(b \Delta^{\prime}(r) \times \Delta^{\prime \prime}(\delta)\right)$ and also, by [F, 2.10.25], that $\mathcal{H}^{1}(K)<\infty$. As before we can establish a Cauchy formula (with $z^{\prime}$ in place of $z_{n}$ ) for $b T(r)$-in fact this case is simpler that the one in Section 1 because the kernel $1 /\left(z^{\prime}-\alpha\right)$ for $\left|z^{\prime}\right|<r$ is smooth on a neighborhood of the support of $b T(r)$ and therefore the argument for the finiteness almost everywhere of the Cauchy transform is not needed. Specifically we obtain the Cauchy formula (3) (in $z^{\prime}$ ) assuming the hypothesis (2) that the slice $\langle T(r), \pi, \alpha\rangle$ exists.

Assuming that $\langle T(r), \pi, \alpha\rangle$ exists, it follows from the Cauchy formula, as before, that there is a representing measure supported on $K$ for each $z \in \operatorname{supp}\langle T(r), \pi, \alpha\rangle$. Therefore $z \in \hat{K}$, where $\hat{K}$ denotes the polynomial convex hull of $K$. Since $\mathcal{H}^{1}(K)<$ $\infty$, there exist a set of positive measure $Q$ in $b \Delta^{\prime}(r)$ and a positive integer $s$ such that $\pi$ maps exactly $s$ points of $K$ to each point of $Q$. By an argument of Bishop (see [A1, Lemma 2]), $V \equiv \hat{K} \cap\left(\Delta^{\prime}(r) \times \Delta^{\prime \prime}(\delta)\right)$ is an analytic cover with (at most) $s$ sheets over $\Delta^{\prime}(r)$. Thus $V$ is a 1 -dimensional subvariety of $\Delta$ such that $z \in V$ for each $z \in \operatorname{supp}\langle T(r), \pi, \alpha\rangle$. This completes the proof of Lemma 12 for $k=1$.

For the proof of step (iii) we need a simple Hartogs type lemma.

Lemma 13. Let $\Delta$ be an open polydisc in $\mathbb{C}^{s}$ with $s>1$ and let $\mathcal{D}$ be a subset of $\Delta$ of full $\mathcal{L}^{2 s}$-measure. Let $F: \mathcal{D} \rightarrow \mathbb{C}$ be a bounded $\mathcal{L}^{2 s}$-measurable function with the following extension property: if $\alpha=\left(\alpha_{1}, \alpha_{2}, \ldots, \alpha_{s}\right) \in \mathcal{D}$, then for all $j$, $1 \leq j \leq s$, there exist an analytic function $f_{\alpha}$ of $(s-1$ variables $)$ on the slice $L_{\alpha, j} \equiv\left\{z \in \Delta: z_{j}=\alpha_{j}\right\}$ such that $F=f_{\alpha}, \mathcal{L}^{2(s-1)}$-almost everywhere on $L_{\alpha, j}$. Then there exists an analytic function $\tilde{F}$ on $\Delta$ such that $\tilde{F}=F, \mathcal{L}^{2 s}$-almost everywhere on $\mathcal{D}$.

Remark. This lemma is quite elementary - the Hartogs type results for meromorphic functions employed by Shiffman [S] lie much deeper.

Proof of Lemma 13. The hypotheses ensure that $\bar{\partial} F=0$ in $\Delta$ in the sense of distributions.

Step (iii). We prove Lemma 12 for $T \in \mathcal{R}_{(k, k)}^{\text {loc }}(\Omega)$ with $d T=0$, assuming, by induction, the Theorem for cases $j<k$. Our previous argument, in Lemmas 9,10 and 11, that $X$ has locally finite $\mathcal{H}^{2 k}$-measure is again valid - note that this argument used induction on the result of our Theorem, but not the Harvey-Shiffman theorem. 
Let $T^{\prime}$ be the positive current in $\mathcal{R}_{(k, k)}^{\text {loc }}(\Delta)$ associated to $T \mid \Delta$ (see the proofs of Lemmas 7 and 11); at this point we cannot assert that $T^{\prime}$ is closed. Then for $\mathcal{L}^{2 k}$ almost all $z^{\prime} \in \Delta^{\prime}$, the slices $\left\langle T \mid \Delta, \pi, z^{\prime}\right\rangle$ and $\left\langle T^{\prime}, \pi, z^{\prime}\right\rangle$ have the same support - set $m\left(z^{\prime}\right)$ equal to the number of points, counting positive multiplicity, in this common support. We claim that $m$ is a measurable function. Note that any slice of $T \mid \Delta$ has its support in $X_{0} \equiv X \cap \Delta$. Hence $\pi$ maps the support of $T^{\prime}$ properly to $\Delta^{\prime}$ and so $\pi_{*}\left(T^{\prime}\right) \in \mathcal{R}_{(k, k)}^{\text {loc }}\left(\Delta^{\prime}\right)$ and therefore $\pi_{*}\left(T^{\prime}\right)=g\left(z^{\prime}\right)\left[\Delta^{\prime}\right]$ for a measurable non-negative integer valued function $g$ on $\Delta^{\prime}$. It follows from the definition of slicing and the positivity of $T^{\prime}$ that $g\left(z^{\prime}\right)=m\left(z^{\prime}\right)$ for $\mathcal{L}^{2 k}$-almost all $z^{\prime} \in \Delta^{\prime}$. This gives the claim. By $[\mathrm{F}, 4.3 .2]$ we have $\int_{\Delta^{\prime}} m\left(z^{\prime}\right) d \mathcal{L}^{2 k} z^{\prime} \leq M\left(T \mid \Delta^{\prime}\right)<\infty$. It follows that $m\left(z^{\prime}\right), \infty$ a.e. and thus there exists a set $A_{1}$ of positive $\mathcal{L}^{2 k}$-measure in $\Delta^{\prime}$ and a non-negative integer $N$ such that $m\left(z^{\prime}\right)=N$ for all $z^{\prime} \in A_{1}$.

By Fubini's theorem there exists a set of positive $\mathcal{L}^{2}$-measure $A_{2} \subseteq\{\lambda \in \mathbb{C}$ : $|\lambda|<r\}$ such that $\left\{z^{\prime} \in A_{1}: \pi_{k}^{\prime}\left(z^{\prime}\right)=\alpha\right\}$ has positive $\mathcal{H}^{2 k-2}$-measure for all $\alpha \in A_{2}$. We may also assume that the slice $\left\langle T \mid \Delta, \pi_{k}, \alpha\right\rangle$ exists for all $\alpha \in A_{2}$. By the induction hypothesis, these slices are holomorphic $(k-1)$-chains with supports in $X_{0}$ and therefore the restriction of $\pi$ to this support is a proper map to $\Delta^{\prime}$ and consequently these supports are branched analytic covers of $\left\{z^{\prime} \in \Delta^{\prime}: \pi_{k}\left(z^{\prime}\right)=\alpha\right\}$. We claim that this is a branched cover of order $N$, counting multiplicity on the support as well as the multiplicity of the map, for $\mathcal{L}^{2}$-almost all $\alpha \in A_{2}$. For the projection $\pi_{J}$ where $J=\{1,2, \ldots, k-1\}$ we have, by Federer's theorem on iterated slicing $[\mathrm{F}, 4.3 .5]$, that

$$
\left\langle T \mid \Delta, \pi, z^{\prime}\right\rangle=\left\langle\left\langle T \mid \Delta, \pi_{k}, \alpha\right\rangle, \pi_{J}, w\right\rangle
$$

for $\mathcal{L}^{2 k}$-almost all $z^{\prime}=(w, \alpha) \in \Delta^{\prime} \subseteq \mathbb{C}^{k-1} \times \mathbb{C}$. Therefore for $\mathcal{L}^{2}$-almost all points $\alpha \in A_{2}$, (10) holds for $\mathcal{L}^{2 k-2}$-almost all $w \in \mathbb{C}^{k-1}$ such that $\|w\|_{\infty}<r$. Fix such an $\alpha$. Since $\left\langle T \mid \Delta, \pi_{k}, \alpha\right\rangle$ has support which is a branched cover of some order, say $s$, counting multiplicity as above, it follows that the support of the right-hand side of (10) has $s$ points for $\mathcal{L}^{2 k-2}$-almost all $w \in \mathbb{C}^{k-1}$ such that $\|w\|_{\infty}<r$. . Therefore $m\left(z^{\prime}\right)=s$ with $z^{\prime}=(w, \alpha)$ for $\mathcal{L}^{2 k-2}$-almost all $w$ such that $\|w\|_{\infty}<r$. As $\alpha \in A_{2}$, we conclude that $s=N$, as claimed. Thus we have shown that $m\left(z^{\prime}\right)=N$ for $\mathcal{L}^{2 k}$-almost all points $z^{\prime} \in A_{3} \equiv\left(\pi_{k}^{\prime} \mid \Delta^{\prime}\right)^{-1}\left(A_{2}\right) \subseteq \Delta^{\prime}$.

Now fix $j$ with $1 \leq j<k$. Suppose that the slice $\left\langle T \mid \Delta, \pi_{j}, \lambda\right\rangle$ exists. Then its support is a branched cover of $\left\{z^{\prime} \in \Delta^{\prime}: \pi_{j}^{\prime}\left(z^{\prime}\right)=\lambda\right\}$ of some integer order. Since $\left\{z^{\prime} \in \Delta^{\prime}: \pi_{j}^{\prime}\left(z^{\prime}\right)=\lambda\right\}$ meets $A_{3}$ in a set of positive $\mathcal{H}^{2 k-2}$-measure for all $\lambda$, it follows as in the last paragraph that the support of $\left\langle T \mid \Delta, \pi_{j}, \lambda\right\rangle$ is a branched cover of order $N$. By repeating the argument of the last paragraph (now with $k$ replaced with $j$ and with $A_{2}$ being a set of full $\mathcal{L}^{2}$-measure in $\left.\{\lambda \in \mathbb{C}:|\lambda|<r\}\right)$ we conclude that $m\left(z^{\prime}\right)=N$ for $\mathcal{L}^{2 k}$-almost all $z^{\prime} \in \Delta^{\prime}$. Summarizing, we have the following.

(i) For $1 \leq j<k$ there is a set $E_{j} \subseteq\{\lambda \in \mathbb{C}:|\lambda|<r\}$ of $\mathcal{L}^{2}$-measure zero such that the slice $\left\langle T \mid \Delta, \pi_{j}, \alpha\right\rangle$ exists and has a support which is a branched cover of order $N$ for all $\alpha \in\{\mathbb{C}:|\lambda|<r\} \backslash E_{j}$. By symmetry we also have a corresponding set for $j=k$.

(ii) There exists a set $Q \subseteq \Delta^{\prime}$ of $\mathcal{L}^{2 k}$ - measure zero such that for all $z^{\prime} \in \Delta^{\prime} \backslash Q$, the slice $\left\langle T \mid \Delta, \pi, z^{\prime}\right\rangle$ exists and its support, counting multiplicity, contains $N$ points.

Recall that (10) holds except for a set $Q_{k}$ of points $z^{\prime}=(w, \alpha) \in \Delta^{\prime}$ of $\mathcal{L}^{2 k}$ measure zero. We set $\mathcal{N}_{k}=Q \cup Q_{k} \cup \pi^{\prime-1}{ }_{k}\left(E_{k}\right) ; \mathcal{N}_{k}$ is a subset of $\Delta^{\prime}$ of $\mathcal{L}^{2 k}$-measure 
zero. Moreover by (10), if $z^{\prime}=(w, \alpha) \in \Delta^{\prime} \backslash \mathcal{N}_{k}$, the $N$ points of $\operatorname{supp}\left\langle T \mid \Delta, \pi, z^{\prime}\right\rangle$ are exactly the $N$ points of $\operatorname{supp}\left\langle T \mid \Delta, \pi_{k}, \alpha\right\rangle$ (= a branched analytic cover) over $z^{\prime}$. Let $\mathcal{N}_{j}$ be the analogous $\mathcal{L}^{2 k}$-null set for $1 \leq j<k$ and put $\mathcal{N}=\bigcup_{j=1}^{k} \mathcal{N}_{j}$. Finally set $\mathcal{D}=\Delta^{\prime} \backslash \mathcal{N}$. Then $\mathcal{D}$ has $\mathcal{L}^{2 k}$-full measure in $\Delta^{\prime}$. By its construction, $\mathcal{D}$ has the following property. Let $z^{\prime} \in \mathcal{D}$. Then (10) applies and the $N$ points in $\operatorname{supp}\left\langle T \mid \Delta, \pi, z^{\prime}\right\rangle$ are the $N$ points in $\operatorname{supp}\left\langle\left\langle T \mid \Delta, \pi_{k}, \pi_{k}\left(z^{\prime}\right)\right\rangle, \pi_{J}, \pi_{J}\left(z^{\prime}\right)\right\rangle$ and also the analogous statements with $k$ replaced with $j, 1 \leq j<k$, hold.

For each point $z^{\prime} \in \mathcal{D}$ there are $N$ points $\left(w_{1}\left(z^{\prime}\right), w_{2}\left(z^{\prime}\right), \ldots, w_{N}\left(z^{\prime}\right)\right)$ in the support of $\left\langle T \mid \Delta, \pi, z^{\prime}\right\rangle$. Let $h$ be a polynomial in $\mathbb{C}^{k}$. Consider the function $\tilde{h}\left(z^{\prime}\right)=$ $\sum_{j=1}^{N} h\left(w_{j}\left(z^{\prime}\right)\right)$ on $\Delta^{\prime}$. We claim that this is an $\mathcal{L}^{k}$-measurable function on $\mathcal{D}$ and therefore on $\Delta^{\prime}$. Indeed $\tilde{h}\left(z^{\prime}\right)=\left\langle T, \pi, z^{\prime}\right\rangle(h)$ for $\mathcal{L}^{k}$-almost all $z^{\prime} \in \Delta^{\prime}$ and the right-hand side is measurable by $[\mathrm{F}, 4.3 .2]$. This gives the claim. Replacing $h$ by $h^{s}$ for a positive integer $s$ gives the measurability of the power sums of $h$. It follows that, for every symmetric polynomial $\sigma\left(X_{1}, X_{2}, \ldots, X_{N}\right)$, that $z^{\prime} \mapsto$ $\sigma\left(w_{1}\left(z^{\prime}\right), w_{2}\left(z^{\prime}\right), \ldots, w_{N}\left(z^{\prime}\right)\right)$ is measurable on $\mathcal{D}$.

Let $f$ be a polynomial in $\mathbb{C}^{n}$. Define, for each $z^{\prime} \in \mathcal{D}$,

$$
P\left(Z, f, z^{\prime}\right)=\prod_{j=1}^{N}\left(Z-f\left(w_{j}\left(z^{\prime}\right)\right)\right),
$$

where $Z$ is an indeterminant and $\left\{w_{1}\left(z^{\prime}\right), w_{2}\left(z^{\prime}\right), \ldots, w_{N}\left(z^{\prime}\right)\right\}$ are the $N$ points of the support of $\left\langle T \mid \Delta, \pi, z^{\prime}\right\rangle$. The coefficients of the polynomial $P$ in $Z$ are symmetric functions of the values of $f$ in $\Delta$ and so are bounded functions. By the previous paragraph these functions are also measurable. We claim that each of these functions on $\mathcal{D}$ satisfies the extension hypothesis of Lemma 13. Indeed, suppose that $z^{\prime} \in \mathcal{D}$. Then for each $j, 1 \leq j \leq k$, the support of the slice $\left\langle T \mid \Delta, \pi_{j}, \alpha\right\rangle$, where $\alpha=\pi_{j}^{\prime}\left(z^{\prime}\right)$, is a branched cover over $L_{\alpha, j} \equiv\left\{\zeta \in \Delta^{\prime}: \pi_{j}(\zeta)=\alpha\right\}$. The associated symmetric functions of this branched cover over $L_{\alpha, j}$ are holomorphic on $L_{\alpha, j}$ and moreover, by the construction of $\mathcal{D}$, these symmetric functions agree with the coefficients of $P$ at almost every point of $L_{\alpha, j} \cap \mathcal{D}$. This gives the claim. By Lemma 13, the coefficients of $Z$ in $P$ are (essentially) holomorphic on $\Delta^{\prime}$. We denote the polynomial, with these holomorphic coefficients on $\Delta^{\prime}$, by $\tilde{P}\left(Z, f, z^{\prime}\right)$. Thus $\tilde{P}\left(Z, f, z^{\prime}\right)$ is a polynomial of degree $N$ in $Z$ with coefficients holomorphic in $z^{\prime} \in \Delta^{\prime}$.

We claim that $g(z) \equiv \tilde{P}\left(f(z), f, z^{\prime}\right)\left(\right.$ a holomorphic function of $\left.z=\left(z^{\prime}, z^{\prime \prime}\right) \in \Delta\right)$ vanishes on the support of the slice $\left\langle T \mid \Delta, \pi_{j}, \alpha\right\rangle$ for $\mathcal{L}^{2}$-almost all $\alpha$ with $|\alpha|<r-$ clearly this holds if

$$
\mathcal{H}^{2 k-2}\left(S_{j}(\alpha)\right)=\mathcal{H}^{2 k-2}\left(\left\{\zeta \in \Delta^{\prime}: \pi_{j}^{\prime}(\zeta)=\alpha\right\}\right)
$$

where $S_{j}(\alpha)=\left\{\zeta \in \mathcal{D}: \pi_{j}^{\prime}(\zeta)=\alpha\right\}$. Since $\mathcal{D}$ has full $\mathcal{L}^{2 k}$-measure in $\Delta^{\prime}$, it follows by Fubini's theorem, that (11) holds for $\mathcal{L}^{2}$-almost all $\alpha$ with $|\alpha|<r$. If (11) holds, then $g(z)=0$ if $z=\left(z^{\prime}, z^{\prime \prime}\right) \in \operatorname{supp}\left\langle T \mid \Delta, \pi_{j}, \alpha\right\rangle$ and $z^{\prime} \in \mathcal{D}$. Since such $z$ are dense in the variety $\operatorname{supp}\left\langle T \mid \Delta, \pi_{j}, \alpha\right\rangle$, we conclude that $g$ vanishes on this variety. This gives the claim. Conversely if $\tilde{P}\left(f(z), f, z^{\prime}\right)=0$ for $z^{\prime} \in \Delta^{\prime}$, then $f(z)$ is one of the $N$ zeros (counting multiplicity) of the polynomial $\tilde{P}\left(Z, f, z^{\prime}\right)$ where $z=\left(z^{\prime}, z^{\prime \prime}\right)$.

Now we apply this construction to the $n-k$ (polynomial) functions $\pi_{\ell}$ for $k+1 \leq$ $\ell \leq n$ to get $n-k$ holomorphic functions $g_{\ell}(z) \equiv \tilde{P}\left(\pi_{\ell}(z), \pi_{\ell}, z^{\prime}\right)$ on $\Delta$. Let $V$ be the common zero set in $\Delta$ of these $n-k$ functions $\left\{g_{\ell}\right\}$. The zero set of each 
$g_{\ell}$ contains the support of the slices $\left\langle T \mid \Delta, \pi_{j}, \alpha\right\rangle, 1 \leq j \leq k$, for $\mathcal{L}^{2}$-almost all $\alpha$ and therefore $V$ also contains these supports. In particular the dimension of $V$ is at least equal to $k$. Moreover, by the last paragraph, for fixed $z^{\prime} \in \Delta^{\prime}$, the set $\left\{z=\left(z^{\prime}, z^{\prime \prime}\right) \in \Delta: g_{\ell}(z)=0\right\}$ consists of points whose $z_{\ell}$-coordinate assumes at most $N$ values for $k+1 \leq \ell \leq n$. It follows that the variety $V$ contains at most $N^{n-k}$ points over each $z^{\prime} \in \Delta^{\prime}$. We conclude $V$ has dimension $k$. This completes the proof of step (iii).

\section{REFERENCES}

[A1] H. Alexander, Polynomial approximation and hulls in sets of finite linear measure in $\mathbb{C}^{n}$, Amer. J. Math. 93 (1971), 65- 74. MR 44:1841

[A2] H. Alexander, Ends of varieties, Bull. Soc. math. France 120 (1992),523-547. MR 93k:32012

[ATU] H. Alexander, B.A.Taylor and J.L. Ullman, Areas of projections of analytic sets, Invent. Math. 16 (1972), 335-341. MR 46:2078

[B] E. Bishop, Conditions for the analyticity of certain sets, Mich. Math. J. 11 (1964), 289-304. MR 29:6057

[DO] A. Davie and B. Øksendal, Peak interpolation for some algebras of analytic functions, Pacific. J. Math. 41 (1972), 81-87. MR 46:9394

[F] H. Federer, Geometric Integration Theory, Springer- Verlag, New York, 1969.

[G] T. Gamelin, Uniform Algebras, Prentice-Hall, Englewood Cliffs,1969. MR 53:14137

[H] R. Harvey, Holomorphic Chains and Their Boundaries, Proc. Sym. Pure Math. 30 (1977), 309-382. MR 56:5929

[HL] F.R. Harvey and H.B.Lawson,Jr., Complex analytic geometry and measure theory, Proc. Sym. Pure Math. 44 (1986), 261-274.

[HS] R. Harvey and B. Shiffman, A characterization of holomorphic chains, Ann. Math. 99 (1974), 553-587. MR 50:7572

[K] J. King, The currents defined by analytic varieties, Acta Math. 127 (1971), 185-220. MR 52:14359

[R] W. Rudin, Function Theory in the Unit Ball of $\mathbb{C}^{n}$, Springer-Verlag, New York, 1980. MR 82i: 32002

[S] B. Shiffman, Complete characterization of holomorphic chains of codimension one, Math. Ann. 274 (1986), 233-256. MR 87h:32022

[S2] B. Shiffman, On the removal of singularities of analytic sets, Michigan Math. J. 15 (1968), 111-120. MR 37:464

[W] J. Wermer, Banach Algebras and Several Complex Variables, Second Edition, SpringerVerlag, New York, 1976. MR 52:15021

Department of Mathematics, University of Illinois at Chicago, 851 S Morgan (M/C 249), Chicago, Illinois 60607-7045

E-mail address: hja@uic.edu 